\title{
LECTEURS 2014
}

Le Comité de rédaction remercie les rapporteurs et les lecteurs ayant apporté leur concours à l'évaluation des articles publiés ou analysés en 2014 (liste au 30 septembre).

AMMERICH Marc, CEA AUBERT Bernard, IRSN

AUBINEAU-LANIECE Isabelle, CEA

BADOT Pierre-Marie, Université Franche Comté

BAGLAN Nicolas, CEA

BAILLY du BOIS Pascal, IRSN

BALDUYCK Sébastien, CHU Toulouse

BARELAUD Bruno, Université Limoges

BARESCUT Jean-Claude, IRSN

BERARD Philippe, CEA

BERTHO Jean-Marc, IRSN

BLANCHARDON Éric, IRSN

BOLCH Wesley, Université Florida (USA)

BORDY Jean Marc, CEA

BOUVILLE André, NIH (USA)

BOYER Patrick, IRSN

CAZOULAT Alain, SPRA

CESARINI Jean-Pierre, Fond ROTSCHILD

CHALLETON de VATHAIRE Cécile, IRSN

CHAMBRETTE Valérie, SFRP

CHAVAUDRA Jean, INSTN

CHEICK THIAM, CEA

CHIAVASSA Sophie, ICO - René Gauducheau

CHOJNACKI Éric, IRSN

CHONSIK LEE, NIH

CLAIRAND Isabelle, IRSN

CORCHO José, BAG (Suisse)

COSSONNET Catherine, IRSN

CROUAIL Pascal, CEPN

DAURES Josiane, CEA

DAVESNE Estelle, IRSN

De VITA Antoine, AREVA

ETARD Cécile, IRSN

FARAH Jad, IRSN

FRANCK Didier, IRSN

GAGNA Gerald, SPRA

GAY Didier, IRSN

GILBIN Rodolphe, IRSN

GONTIER Éric, HIA - Val de Grace

GROETZ Jean-Emmanuel, Université Franche Comté

GSCHWIND Régine, Université Franche Comté

GUERSEN Joël, Hôpital Clermont Ferrand

GUETAT Philippe, CEA

HARTMANN Philippe, EDF

HEMIDY Pierre-Yves, EDF

HUET Christelle, IRSN

LALLEMAND Jeannine, EDF

LAMART Stéphanie, NIH Bethesda (USA)

LAZO Ted, OCDE
Le DIZES-MAUREL Séverine, IRSN

LEBARON-JACOBS Laurence, CEA

LEMAITRE Nathalie, IRSN

LENOIR de CARLAN LoÏc, CEA

LOPEZ Maria Antonia, CIEMAT (Espagne)

MAGNE Isabelle, EDF

MAKOVICKA Libor, Université Franche Comté

MANDUCI Loredana, EAMEA

MANGERET Arnaud, IRSN

MARIE Laurent, IRSN

MARO Denis, IRSN

MAUBERT Henri, CEA

MENECHAL Philippe, ASN

METIVIER Henri, SFRP

MILLION Marc, Landauer

MURITH Christophe, BAG (Suisse)

NGUYEN Duc, IREC (Canada)

NICOLAS Emmanuel, Bureau Veritas

NOSSKE Dietamar, BfS (Allemagne)

OUBEREHIL Allal, TDF

PAQUET François, IRSN

PAUL Didier, CEA

PERRIN Anne, MINATEC

PESENTI-ROSSI David, Hôpital Versailles

PETEGNIEF Yolande, Besançon

PIERRAT Noëlle, Institut Curie

POURCELOT Laurent, IRSN

POURCHER Thierry, CEA

RICARD Marcel, IGR

ROUSSE Carole, ASN

SARRAZIN Thierry, Université Lille

SCANFF Pascale, IRSN

SEVESTRE Bernard, CEA

SEZE de René, INERIS

SOLC Jaroslav, Czech Metrology institute (Czech Republic)

SOUQUES Martine, EDF

TAMPONNET Christian, IRSN

THIERRY-CHEF Isabelle, IARC Lyon

THIRY Yves, Andra

TIRMARCHE Margot, ASN

VAILLANT Ludovic, CEPN

VALERO Marc, ASN

VAN RONGEN Éric, ICNIRP

VARIN Jean-Christophe, AREVA

VECCHIA Paolo, ICNIRP

VEYRET Bernard, Université Bordeaux

VIAL Thierry, CEA

VIVIER Alain, CEA

ZANKL Maria, Helmholtz (Allemagne) 